\title{
Parámetros de calidad de cuatro tipos de formulaciones a base de Trichoderma asperellum y Purpuricillium lilacinum
}

\section{(Quality parametres of four types of formulations based on Trichoderma asperellum and Purpuricillium lilacinum)}

\author{
William Viera ${ }^{1}$, Michelle Noboa ${ }^{2}$, Johana Bermeo ${ }^{3}$, Francisco Báez $^{1}$, Trevor Jackson ${ }^{4}$
}

\begin{abstract}
Resumen:
El género Trichoderma y en menor grado Purpureocillium, han sido estudiados ampliamente por sus propiedades como microorganismos benéficos y su eficacia en el control de enfermedades. Sin embargo, la eficacia biológica, no es la única medida que se considera al momento de formular un microorganismo; la viabilidad, la pureza y la concentración en la formulación, son características importantes para asegurar el éxito en campo. El propósito de este estudio fue probar distintos tipos de formulaciones (gránulos, polvos, líquidos) registrar su comportamiento en el tiempo, utilizando las variables viabilidad, concentración y pureza para seleccionar las mejores formulaciones en función de estos parámetros. Como resultado se obtuvo que los bioformulados a base de $T$. asperellum presentaron mayor estabilidad durante el almacenamiento, siendo más estables las formulaciones sólidas secas en comparación con los productos líquidos. En los formulados para P. lilacinum, se observó que la presentación de polvos mojables muestran la concentración y la estabilidad más alta en el tiempo.
\end{abstract}

Palabras clave: Trichoderma; Purpureocillium; viabilidad; pureza; concentración.

\begin{abstract}
:
The genus Trichoderma and to a lesser extent Purpureocillium, have been studied extensively for their properties as beneficial microorganisms and their effectiveness in disease control. However, biological efficacy is not the only parameter considered when formulating a microorganism; viability, purity and concentration in the formulation are important characteristics to ensure success in the field. The aim of this study was to access different types of formulations (granules, powders, liquids) to record their behavior over time, using the variables viability, concentration and purity to select best formulations based on these parameters. As a result, it was obtained that the bioformulates based on $T$. asperellum showed greater stability during storage, with dry solid formulations being more stable in comparison with liquid products. In terms of the $P$. lilacinum formulations, it was observed that the presentation of wettable powders showed the highest concentration and stability over the time.
\end{abstract}

Keywords: Trichoderma; Purpureocillium; viability; purity; concentration

\section{Introducción}

La llamada revolución verde, iniciada en la década de los años 50 , ha causado un impacto importante y a la vez negativo en el ámbito de la agricultura y el ambiente (Djurfeldt et al., 2005). Como parte de las alternativas para el manejo ambientalmente sostenible de los problemas fitosanitarios de los cultivos, se destaca el uso de hongos benéficos tales como los del género Trichoderma asperellum (Samuels, Lieckf y Nirenberg, 1999)

\footnotetext{
1 Instituto Nacional de Investigaciones Agropecuarias (INIAP), Cutuglahua, Ecuador (willydnpv@hotmail.com).

${ }^{2}$ Instituto Interamericano de Cooperación para la Agricultura (IICA), Quito, Ecuador.

3 Universidad Politécnica Salesiana, Quito, Ecuador.

${ }^{4}$ AgResearch, Lincoln, Nueva Zelanda.
} 
caracterizado y usado en el control biológico por sus cualidades de micoparasitismo, antibiosis y antagonismo de hongos fitopatógenos (Harman, 2005; Masunaka et al., 2011), competencia por los nutrientes y capacidad de promover el crecimiento (Inbar et al., 1994). Algunas cepas de Trichoderma spp., son agentes eficaces de control biológico contra varios patógenos de plantas demostrando resultados positivos en ensayos experimentales (Govindasamy y Balasurbramanuan, 1989; Jayaraj et al., 2006).

La capacidad antagónica de Trichoderma spp., sobre fitopatógenos ocurre principalmente por micoparasitismo, el cual depende de la secreción de complejas enzimas hidrolíticas capaces de degradar la pared de la célula huésped. La actividad antifúngica de la $\alpha-1,3-$ glucanasa (EC 3.2.1.59, enzimas capaces de degradar $\alpha-1,3-$ glucanos y también denominadas mutanasas) se han descrito en Trichoderma harzianum y T. asperellum (Sanz et al., 2004).

Asimismo, Purpureocillium lilacinum (Hywel-Jones y Samson, 2011) es otra especie fúngica que ha demostrado un interesante potencial para ser desarrollado como herramienta de control biológico, debido a su actividad nematicida (Núñez et al., 2012) y entomopatógena (Castillo et al., 2014).

Para la utilización de estos microorganismos como agentes de control biológico es necesario desarrollar sistemas de producción y formulación que permitan contar con productos de la misma o mayor eficacia que los productos químicos; una adecuada y suficiente vida útil es uno de los requisitos para la aceptación y comercialización de un producto biológico. La estabilidad en almacenamiento depende de factores externos como la temperatura, la humedad relativa y el tipo de empaque utilizado, lo que determina el tiempo durante el cual se mantienen las características iniciales del agente biológico y por lo tanto su actividad biocontroladora (Santos et al., 2012).

El uso de formulaciones basadas en microorganismos está creciendo constantemente con la finalidad de reducir el uso de pesticidas (Gerhardson, 2002). Estudios en todo el mundo han desarrollado formulaciones de distinto tipo, algunas puras o mixtas, con uno o más microorganismos benéficos (Arora et al., 2010); en cuanto a la presentación se han formulado gránulos recubiertos, polvos solubles, suspensiones líquidas y emulsiones (Jones y Burges, 1998). Sin embargo, un sustrato ideal no solo debe contener partículas de correctas dimensiones, sino también mantener la integridad estructural durante la preparación para el proceso de producción (López et al., 2015).

El propósito de esta investigación fue evaluar distintos parámetros asociados a la calidad de producto formulado con cepas de T. asperellum y P. lilacinum.

\section{Materiales y Métodos}

Esta investigación se desarrolló en el Laboratorio de Control Biológico de la Estación Experimental Santa Catalina del INIAP (temperatura de $18.6^{\circ} \mathrm{C}$ y humedad relativa de $54 \%)$.

\subsection{Microorganismos}

Los aislamientos de los hongos Trichoderma asperellum y Purpureocillium lilacinum fueron obtenidos del laboratorio de Nematología de la Estación Experimental Litoral Sur, se utilizaron las cepas G8 de $T$. asperellum y ECU-1 de P. lilacinum, las cuales fueron aisladas y cultivadas en cajas de Petri con PDA incubadas por 8 días a $28^{\circ} \mathrm{C}$.

\subsection{Producción masiva de esporas de $T$. asperellum y $P$. Iilacinum}

Para la obtención de conidios secos de los hongos, se realizó la producción masiva utilizando como sustrato arroz pilado de grano entero con una humedad del $25 \%$, contenido en bolsas plásticas y esterilizado a $121^{\circ} \mathrm{C}$ por 15 minutos. La inoculación se realizó con $10 \mathrm{~mL}$ de suspensión de conidios $\left(2,59 \times 10^{9}\right.$ conidios $\left./ \mathrm{mL}\right)$ por cada $100 \mathrm{~g}$ del sustrato 
estéril. Posteriormente las bolsas inoculadas se incubaron a $25{ }^{\circ} \mathrm{C}$ por el lapso de 5 días en el caso de $T$. asperellum y 7 días para $P$. lilacinum.

Para formulaciones en polvo mojable y concentrado emulsionable $100 \mathrm{~g}$ de arroz esporulado se trasvasaron a fundas de papel Kraft para permitir el secado y a continuación se realizó la cosecha de esporas mediante tamizaje. Los conidios secos recuperados se almacenaron en desecadores a $26^{\circ} \mathrm{C}$ para la ganancia de humedad ambiental. Las formulaciones en gránulos dispersables y cubiertos se realizaron mediante lavado directo de $100 \mathrm{~g}$ de sustrato utilizando $40 \mathrm{~mL}$ de agua estéril con Tritón al $0.001 \%$.

\subsection{Formulación de gránulos dispersables}

Para la formulación de T. asperellum se emplearon $43.31 \%$ de caldo madre, $4.08 \%$ de material aglutinante, $52.62 \%$ de material dispersante. Además, en la formulación de $P$. lilacinum se usó $35.18 \%$ de caldo madre, $4.66 \%$ de material aglutinante y $60.16 \%$ de dispersantes. En ambos casos, se utilizó un extrusor manual para formación de gránulos. El material extruido se secó a temperatura ambiente $\left(\approx 15^{\circ} \mathrm{C}\right)$ por 5 días y luego se empacó en bolsas metalizadas selladas al calor.

\subsection{Formulación de gránulos cubiertos}

Se preparó una pasta de recubrimiento conformada por $4 \%$ del agente espesante, $4 \%$ de material transportador líquido y $92 \%$ de caldo madre. Esta se mezcló en proporción 1:3 con el soporte mineral sólido y cuando este estuvo completamente húmedo, se agregó un material secante en polvo en proporción 1:1 con respecto al soporte utilizado. Finalmente se adicionó otra cantidad igual de material secante hasta obtener flujo libre en los gránulos cubiertos, los cuales se colocaron en bandejas para acelerar su secado y posteriormente se empacaron en bolsas metalizadas selladas al calor. El proceso fue realizado en la misma proporción para ambas especies de hongos.

\subsection{Formulación de polvos mojables}

Para el caso de T. asperellum se mezcló $45 \%$ del material transportador, $45 \%$ del agente dispersante, $7 \%$ del aglutinante y $3 \%$ de esporas secas. Para $P$. lilacinum se combinó $42 \%$ del material transportador, $42 \%$ del agente dispersante, $6 \%$ del aglutinante y $10 \%$ de esporas secas. Los prototipos una vez formulados fueron empacados en bolsas metalizadas selladas al calor.

\subsection{Formulación de concentrado emulsionable}

Para este prototipo se utilizó aceite mineral como portador líquido. Para $T$. asperellum los porcentajes fueron $99.25 \%$ del aceite, $0.50 \%$ del agente surfactante y $0.25 \%$ de espora seca. Para el caso de P. lilacinum se utilizó $92.55 \%$ de aceite, $5.00 \%$ del agente surfactante y $2.45 \%$ de esporas secas. Las suspensiones oleosas se mezclaron el agente surfactante y la espora seca, luego se agitaron a $150 \mathrm{rpm}$ por 30 minutos y luego por 200 rpm por 15 minutos, finalmente se envasaron en recipientes plásticos y se almacenaron a una temperatura de $6{ }^{\circ} \mathrm{C}$.

\subsection{Estudio de estabilidad en almacenamiento}

En formulaciones sólidas se emplearon 90 muestras empacadas en bolsas de aluminio con cierre hermético de $500 \mathrm{~g}$ de capacidad $(10 \mathrm{~cm} \times 17 \mathrm{~cm})$. En la formulación líquida se utilizaron 30 frascos plásticos (transparentes de $500 \mathrm{~mL}$ ) Las muestras se almacenaron en una estantería ubicada en un cuarto con temperatura $16 \pm 2{ }^{\circ} \mathrm{C}$. El estudio fue diseñado completamente al azar, con arreglo factorial, la unidad experimental fue de 
una bolsa o frasco por tipo de formulación y por especie de hongo, con 30 réplicas por cada tratamiento, con un total de 240 unidades. El muestreo se realizó en bolsas independientes, descartando luego la bolsa pero utilizando material del mismo lote para la evaluación en el tiempo. Las evaluaciones se realizaron cada 15 días durante dos meses se evaluaron los parámetros que se describen a continuación:

\subsection{Concentración}

Se tomaron 30 muestras de $1 \mathrm{~g} \mathrm{o} 1 \mathrm{~mL}$ de cada formulación, los cuales fueron reconstituidos utilizando $9 \mathrm{~mL}$ de una solución de Tritón X-100 al $0.1 \%$. Se realizaron diluciones seriadas desde $10^{-1}$ hasta $10^{-6}$, de las tres primeras diluciones se tomaron $20 \mu \mathrm{L}$ y se colocaron en cámara Neubauer para determinar la concentración de conidios utilizando un microscopio óptico (Olympus, BX-41). Las evaluaciones se realizaron en tres ocasiones, la primera el día del empacado del bioformulado, la segunda a los 30 días y la tercera a los 60 días.

\subsection{Viabilidad}

Se evaluó la viabilidad expresada como la capacidad de germinación de los conidios en los formulados. Se sembró $100 \mu \mathrm{l}$ de diluciones seriadas $10^{-6}, 10^{-5}$ y $10^{-4}$ en medio de cultivo PDA con adición de ácido láctico $\left(320 \mu \mathrm{L} \mathrm{L}^{-1}\right)$ y Tritón X-100 al $0,1 \%$. Las cajas de Petri fueron incubadas $25^{\circ} \mathrm{C}$ por un período de 4 días para formulaciones a base de $T$. asperellum y 6 días para el caso de $P$. lilacinum, momento en que se realizó el recuento de unidades formadoras de colonia (UFC). Las evaluaciones se realizaron el día de envasado del producto y a los 15, 30, 45 y 60 días de almacenamiento. El cálculo de viabilidad se realizó utilizando la siguiente fórmula:

Viabilidad $(\mathrm{UFC} / \mathrm{g})=$ promedio de colonias del hongo $\mathrm{x}$ factor de dilución $\mathrm{x}$ factor de siembra de $10(1000 \mu \mathrm{L} / 100 \mu \mathrm{L})$

\subsection{Pureza}

Con las siembras realizadas en las cajas Petri para la evaluación de la viabilidad de los microorganismos, se contabilizó el número de colonias de hongo benéfico en cada caja Petri, en las diluciones de $10^{-4}, 10^{-5}$ y $10^{-6}$. Se realizaron 4 evaluaciones en períodos de 15 días, la primera evaluación se realizó el día del empaquetado o envasado del bioformulado, el cálculo se efectuó aplicando la siguiente fórmula (Castro, 2013).

Pureza $(\%)=$ Concentración de Trichoderma (número de colonias $\mathrm{x}$ el factor de dilución) / concentración de microorganismos totales (concentración del benéfico + concentración de los contaminantes) x 100

\subsection{Análisis estadístico}

Mediante un diseño completamente al azar con arreglo factorial $4 \times 2$ (cuatro tipos de formulación y dos especies de hongos antagonistas) con 30 observaciones por tratamiento. El análisis de los bioformulados, incluyó los siguientes parámetros: (I) concentración de conidios en el tiempo, (II) viabilidad en el tiempo, y (III) porcentaje de pureza de las formulaciones en el tiempo. Cada uno de estos parámetros fue analizado estadísticamente de manera individual con base a la especie de microorganismo, $T$. asperellum y $P$. lilacinum, respectivamente. En todas las variables evaluadas se se empleó el análisis estadístico de la prueba no paramétrica de Kruskall Wallis. La comparación de medias para porcentaje de pureza, concentración y viabilidad se realizó con el test de Wilcoxon Rank, todos los análisis estadísticos se llevaron a cabo utilizando el software R versión 3.2.2. 


\section{Resultados y Discusión}

\subsection{Viabilidad del formulado}

Con respecto a $T$. asperellum el test de Kruskal Wallis para datos no paramétricos determinó que no hubo significancias estadísticas para el factor día ( $d f=597, p=0.824$ ), por lo que se infiere que la viabilidad se mantuvo estable en el tiempo, sin embargo, en lo que respecta al factor tipo de formulación se observó que existen diferencias estadísticas significativas $(\mathrm{df}=597, \mathrm{p}<0.001)$ y que fueron los formulados de gránulos solubles y cubiertos aquellos que mantuvieron sus esporas viables en mayor cantidad respecto a las formulaciones líquidas y en polvo (Tabla 1). Sin embargo, Kumar et al. (2014) menciona que las formulaciones a base de aceite son adecuadas para ser utilizadas en aspersiones foliares por tener vida útil prolongada por lo que este tipo de bioformulado es muy utilizado en la agricultura.

En las formulaciones de $P$. lilacinum se observó un comportamiento diferente, observándose diferencias altamente significativas para el factor día $(\mathrm{df}=597, \mathrm{p}<0.001)$ y para el factor tipo de formulación ( $d f=597, p<0.001$ ). Se observa que a los 60 días la germinación disminuye en todas las formulaciones independientemente del tipo. Además se observa que la formulación líquida fue aquella que presentó los valores más bajos y que su comportamiento en el tiempo fue más variable (Tabla 1); resultado opuesto al encontrado por Cardona et al. (2014) quien reportó que la formulación líquida de Purpureocillium sp. (cepa comercial) mantuvo una viabilidad estable, lo que indicaría que hay una variación entre cepas de este hongo. Además, Barra et al. (2018) indicó que tipo de sustrato utilizado como portador para un formulación tiene efecto sobre la viabilidad de $P$. lilacinum, resultado que también se produjo en este estudio.

Tabla 1. Cantidad de conidios (UFC $\mathrm{g}^{-1}$ ) de $T$. asperellum $\mathrm{G} 8$ y $P$. lilacinum PL-ECU-1 viables en formulaciones almacenadas durante 60 días a $25^{\circ} \mathrm{C}$.

\begin{tabular}{|c|c|c|c|c|c|c|}
\hline \multirow{2}{*}{ Especies } & \multirow{2}{*}{ Formulación } & \multicolumn{5}{|c|}{ Tiempo de almacenamiento (días) } \\
\hline & & 0 & 15 & 30 & 45 & 60 \\
\hline \multirow{4}{*}{$\begin{array}{l}\text { Trichoderma } \\
\text { asperellum } \\
\text { G8 }\end{array}$} & Gránulos solubles & $1.6 \times 10^{8}$ & $1.83 \times 10^{8}$ & $1.7 \times 10^{8}$ & $1.73 \times 10^{8}$ & $1.7 \times 10^{8}$ \\
\hline & Gránulos cubiertos & $1.6 \times 10^{8}$ & $1.6 \times 10^{8}$ & $1.7 \times 10^{8}$ & $1.6 \times 10^{8}$ & $1.6 \times 10^{8}$ \\
\hline & Polvos mojables & $4.2 \times 10^{7}$ & $4.7 \times 10^{7}$ & $4.2 \times 10^{7}$ & $4.9 \times 10^{7}$ & $3.6 \times 10^{7}$ \\
\hline & Líquido & $3.0 \times 10^{6}$ & $2.8 \times 10^{6}$ & $2.8 \times 10^{6}$ & $2.6 \times 10^{6}$ & $2.4 \times 10^{6}$ \\
\hline \multirow{4}{*}{$\begin{array}{l}\text { Purpureocilium } \\
\text { lilacinum } \\
\text { PL-ECU-1 }\end{array}$} & Gránulos solubles & $3.1 \times 10^{8}$ & $2.4 \times 10^{8}$ & $2.2 \times 10^{8}$ & $2.3 \times 10^{8}$ & $1.1 \times 10^{8}$ \\
\hline & Gránulos cubiertos & $3.2 \times 10^{8}$ & $2.1 \times 10^{8}$ & $2.0 \times 10^{8}$ & $2.0 \times 10^{8}$ & $1.2 \times 10^{8}$ \\
\hline & Polvos mojables & $1.7 \times 10^{8}$ & $1.3 \times 10^{8}$ & $1.7 \times 10^{8}$ & $1.7 \times 10^{8}$ & $1.1 \times 10^{8}$ \\
\hline & Líquido & $7.4 \times 10^{6}$ & $9.1 \times 10^{6}$ & $8.1 \times 10^{6}$ & $2.0 \times 10^{7}$ & $1.6 \times 10^{7}$ \\
\hline
\end{tabular}

Si bien las formulaciones presentaron valores fluctuantes estos son mínimos, no varían en el exponencial de viabilidad, por lo que se consideran estables en el tiempo de dos meses (Tabla 1). De acuerdo con estudios realizados por Ruíz, Gómez y Villamizar (2015) las pérdidas de viabilidad de las formulaciones almacenadas a temperatura ambiente son del $5 \%$ formulaciones de polvos mojables y del $17,3 \%$ en formulaciones granuladas. En el presente estudio se observa que las formulaciones de polvos mojables tuvieron una pérdida del $14 \%$ en su viabilidad para Trichoderma asperellum y un $35 \%$ para $P$. lilacinum.

En general, Kumar et al. (2014) menciona que los hongos antagonistas que se multiplican usando un portador orgánico tienen una vida útil más larga que los formulados con portadores inertes o inorgánicos. 


\subsection{Concentración del formulado}

Con respecto a $T$. asperellum la prueba de Kruskal - Wallis determinó que hubo diferencias significativas para el factor día $(\mathrm{df}=237, p<0.001)$ y para el factor tipo de formulación ( $d f=237, p<0.001)$. Kumar et al. (2014) menciona que la concentración mínima de Trichoderma debe ser de $2 \times 10^{6}$ UFC g-1 en un medio selectivo, los resultados mostrados en la Tabla 2 indican que todas las formulaciones alcanzaron este nivel de concentración.

En las formulaciones de $P$. lilacinum también se observaron diferencias altamente significativas para el factor día $(\mathrm{df}=237, \mathrm{p}<0.001)$ y para el factor tipo de formulación $(\mathrm{df}=237, p<0.001)$. Las formulaciones de gránulos cubiertos resultaron ser las más estables en el tiempo tanto en $T$. asperellum como en $P$. lilacinum.

Como se observa en la Tabla 2, las formulaciones presentaron valores fluctuantes pero estos estos son mínimos, no varían en el exponencial de la concentración en el tiempo. Para las formulaciones de gránulos solubles de $T$. asprellum se observa una disminución en la concentración de $26 \%$ en el lapso de dos meses. Sin embargo, para en las formulaciones de $P$. lilacinum se observa un incremento en la concentración del $16 \%$ lo cual indica que probablementen existió humedad en el almacenamiento de las formulaciones y a su vez esto produjo un incremento en la tasa reproductiva del hongo. Otra probable causa del aumento de la concentración se pudo deber a la variación de concentración debido al muestreo aleatorio para seleccionar las bolsas dentro del lote.

Tabla 2. Concentración de los conidios (UFC $\mathrm{g}^{-1}$ ) de $T$. asperellum G8 y $P$. lilacinum PL-ECU-1 en formulaciones almacenadas durante 60 días.

\begin{tabular}{llll} 
& & \multicolumn{2}{l}{$\begin{array}{l}\text { Tiempo de } \\
\text { almacenamiento } \\
\text { (días) }\end{array}$} \\
\cline { 2 - 4 } & Formulación & 0 & 60 \\
\hline \multirow{2}{*}{ T. asperellum } & Gránulos solubles & $3.4 \times 10^{8}$ & $2.5 \times 10^{8}$ \\
G8 & Gránulos cubiertos & $1.0 \times 10^{8}$ & $3.1 \times 10^{8}$ \\
& Polvos mojables & $1.7 \times 10^{8}$ & $5.6 \times 10^{8}$ \\
& Líquido & $2.6 \times 10^{7}$ & $1.9 \times 10^{7}$ \\
\hline \multirow{2}{*}{ P. lilacinum } & Gránulos solubles & $8.60 \times 10^{8}$ & $1.0 \times 10^{9}$ \\
PL-ECU-1 & Gránulos cubiertos & $6.4 \times 10^{8}$ & $6.4 \times 10^{8}$ \\
& Polvos mojables & $6.7 \times 10^{8}$ & $6.4 \times 10^{8}$ \\
& Líquido & $3.5 \times 10^{9}$ & $1.0 \times 10^{8}$ \\
\hline
\end{tabular}

\subsection{Pureza del formulado}

Cuando los productos fueron almacenados y se contabilizó cada 15 días el porcentaje de pureza. En $T$. asperellum no se evidenció un incremento del porcentaje de contaminantes para el factor formulación, debido a que no se obtuvieron diferencias estadísticas significativas $(\mathrm{df}=597, \mathrm{p}=0.06)$. Sin embargo, se presentaron diferencias significativas para el factor tiempo ( $d f=597, p=0.002)$, lo cual indica que el número de días de almacenamiento influye en el porcentaje de contaminación.

Con respecto a $P$. lilacinum no se observaron diferencias estadísticas significativas para ninguno de los dos factores tiempo y tipo de formulación ( $d f=597, p=0.07)$ y $(d f=597$, $\mathrm{p}=0.225$ ) respectivamente, lo cual indica que el tiempo de almacenamiento y el tipo de formulación hasta los 60 días no afecta de manera negativa a la pureza. Barra et al. (2018) encontró que en formulaciones con sustrato de partículas el porcentaje de pureza estaba alrededor de $99 \%$, mientras que en formulación de polvo la pureza era baja con valores desde 23 a $75 \%$; sin embargo, en nuestro estudio todos los valores de pureza fueron sobre el $98 \%$ para todos los tipos de formulación (Tabla 3).

Se obtuvieron valores de contaminación no superiores al $2 \%$ en todas las formulaciones, tanto para $T$. asperellum como para P. lilacinum (Tabla 3). Los valores que 
se obtuvieron están acordes con los parámetros de calidad que sugieren Marín et al. (2002), quienes señalan que las formulaciones comerciales tienen una pureza adecuado cuando esta excede el $80 \%$. Estos porcentajes mínimos de contaminación al igual que la fluctuación en los valores se pueden deber a contaminantes ambientales que pudieron ingresar al producto durante el proceso de formulación ya que no se realizan en condiciones de esterilidad absoluta.

Tabla 3. Porcentajes de pureza en formulaciones de bioplaguicidas de $T$. asperellum $G 8$ y $P$. lilacinum ECU-1 en formulaciones almacenados durante 60 días a $16 \pm 2{ }^{\circ} \mathrm{C}$.

\begin{tabular}{lllllll}
\hline \multirow{2}{*}{ Cepa } & \multirow{2}{*}{ Formulación } & \multicolumn{6}{l}{ Tiempo de almacenamiento (días) } \\
\cline { 3 - 7 } & & 1 & 15 & 30 & 45 & 60 \\
\hline \multirow{2}{*}{$\begin{array}{l}\text { asperellum } \\
\text { G8 }\end{array}$} & Gránulos solubles & 98.57 & 98.45 & 99.95 & 98.81 & 99.81 \\
& Gránulos & & & 98.71 & 98.84 & 99.78 \\
& cubiertos & 99.72 & 99.86 & 98.71 & 99.70 & 100.00 \\
& Polvos mojables & 99.17 & 98.87 & 99.49 & 99.70 & 99.36 \\
\hline \multirow{4}{*}{ P. lilacinum } & Líquido & 98.74 & 98.43 & 98.50 & 98.59 & 99.00 \\
PL-ECU-1 & Gránulos solubles & 98.99 & 100.00 & 98.86 & 99.62 & 100.00 \\
& cubiertos & 99.88 & 98.77 & 98.55 & 98.89 & 99.98 \\
& Polvos mojables & 99.48 & 98.64 & 99.78 & 99.62 & 99.89 \\
& Líquido & 99.88 & 99.94 & 98.71 & 99.94 & 99.97 \\
\hline
\end{tabular}

En general, se puede apreciar que realizar un control de calidad durante el almacenamiento del producto, tomando en cuenta los parámetros de viabilidad, concentración y pureza es obligatorio para garantizar la calidad del formulado, puesto que cuando el control de calidad es inadecuado, la contaminación microbiana del producto final es inevitable. Por esta razón debe reconocerse que los procedimientos de control de calidad pueden ser más complejos y tecnológicamente más exigentes que los propios procedimientos de producción (Jenkins y Grzywacz, 2003).

\section{Conclusiones}

La formulación granulada obtuvo los mejores resultados en concentración de esporas y viabilidad de los microorganismos. Este tipo de formulación fue la más adecuada en las condiciones de evaluación de este estudio. Además, las formulaciones líquidas fueron las menos efectivas debido a los valores bajos de los parámetros mencionados anteriormente. La pureza obtenida en todos los tipos de formulación fue alta, lo que garantiza la calidad del proceso de formulación. Estos resultados son favorables para el posicionamiento comercial de las formulaciones en polvo y gránulos evaluadas en el presente trabajo, ya que soportan bien temperaturas ambientales de $25{ }^{\circ} \mathrm{C}$ no requiriendo cadena de refrigeración para su transporte y almacenamiento.

\section{Bibliografía}

Arora, N., Khare, E., y Maeshwari, E. (2010). Plant growth promoting rhizobacteria: Constraints in bioformulation, commercialization, and future strategies. Mycrobiology Monographs, 18(1), 97-116. https://doi.org/10.1007/978-3-642-13612-2_5

Barra, P., Barros, G., Etcheverry, M., y Nesci, A. (2018). Mass production studies in solid substrates with the entomopathogenic fungus, Purpureocillium lilacinum. International Journal of Advance Agricultural Research, 6(1), 78-84.

Cardona, N., Borrego, D., Fernández, E., Sánchez, J., Cardona, V., y Montoya, G. (2014). Evaluación microbiológica y patogenicidad de una bioformulacion líquida del hongo 
Purpureocillium sp. (cepa UdeA 0109) sobre estadios de Meloidogyne incognitajavanica. Biotecnología Aplicada, 31(1), 204-209.

Castillo, D., Zhu, K., Ek, M., y Sword, G. (2014). The entomopathogenic fungal endophytes Purpureocillium lilacinum (formerly Purpureocillium lilacinus) and Beauveria bassiana negatively affect cotton aphid reproduction under both greenhouse and field conditions. Plos One, 9(8), e103891. https://doi.org/10.1371/journal.pone.0103891

Djurfeldt, G., Holmén, H., Jirstrom, M., y Larsson, R. (2005). The African food crisis: Lessons from the Asian green revolution. Wallingford: CABI Publishing.

Gerhardson, B. (2002) Biological substitutes for pesticides. Trends in Biotechnology, 20(8), 338-343. https://doi.org/10.1016/S0167-7799(02)02021-8

Govindasamy V. y Balasurbramanuan R. (1989). Biological control of groundnut rust, Puccinia arachidis, by Trichoderma harzianum. Journal of plant disease protection. 96: 337-345.

Harman, G. (2005). Overview of mechanisms and uses of Trichoderma spp. Phytopathology, 96(2), 190-194. https://doi.org/10.1094/PHYTO-96-0190

Jayaraj, J., Radhakrishnan, N., y Velazhahan, R. (2006). Development of formulations of Trichoderma harzianum strain M1 for control of damping-off of tomato caused by Pythium aphanidermatum. Archives of Phytopathology and Plant Protection, 39(1), 18. https://doi.org/10.1080/03235400500094720

Jenkins, N., y Grzywacz, D. (2003). "Quality control and production of biological control agents: theory and testing procedures". En: Van Lenteren, J. (Ed.). Towards the standardization of quality control of fungal and viral biological control agents. Silwood Park: CABI Bioscience.

Jones, K., y Burges, D. (1998). "Technology on formulation and application”. En: Burges, D. (Ed). Formulation of Microbial Biopesticides. Dodrecht: Kluwer Academic Publisher.

Kumar, S., Thakur, M., y Rani, A. (2014). Trichoderma: Mass production, formulation, quality control, delivery and its scope in commercialization in India for the management of plant diseases. African Journal of Agricultural Research, 9(53), 38383852. http://doi.org/10.5897/AJAR2014.9061

López, M., Rodríguez, D., y Loera O. (2015). Production of conidia of Beauveria bassiana in solid-state culture: Current status and future perspectives. Critical Reviews in Biotechnology, 35(3),334-341. https://doi.org/10.3109/07388551.2013.857293

Marín, P., y Bustillo, A. (2002). Pruebas microbiológicas y fisicoquímicas para el control de calidad de los hongos entomopatógenos. En: Memorias Curso Internacional TeóricoPráctico sobre entomopatógenos, parasitoides y otros enemigos naturales de la broca del café. Chinchiná: Colombia.

Masunaka, A., Hyakumachi, M., y Takenaka, S. (2011). Plant growth-promoting fungus, Trichoderma koningi suppresses isoflavonoid phytoalexin vestitol production for xolonization on/in the eoots of Lotus japonicus. Microbes and Environments, 26(2), 128-134.

Núñez, M., Carrión, G., Núñez, A., y López, D. (2012). Evaluación de la patogenicidad in vitro de Purpureocillium lilacinum sobre Globodera rostochiensis. Tropical and Subtropical Agrosystems, 15(2), 126-134.

Ruiz, C., Gómez, M., y Villamizar, L. (2015). Prototipo de formulación y atmósfera de empaque para la cepa antagonista Pseudomonas fluorescens Ps006. Revista Colombiana de Biotecnología, 17(2), 95-102. https://doi.org/10.15446/ rev.colomb.biote.v17n2.54282

Santos, A., García, M., Cotes, A. M., y Villamizar, L. (2012). Efecto de la formulación sobre la vida útil de bioplaguicidas a base de dos aislamientos colombianos de Trichoderma koningiopsis Th003 y Trichoderma asperellum Th034. Revista Iberoamericana de Micología, 29(3), 150-156. https://doi.org/10.1016/j.riam.2011.11.002 
Sanz, L., Montero, M., Redondo, J., Llobell, A., \& Monte, E. (2005). Expression of an $\alpha-1$, 3-glucanase during mycoparasitic interaction of Trichoderma asperellum. The FEBS journal, 272(2), 493-499. 